\title{
Influence of the volume ratio of solid phase on carrying capacity of regular porous structure
}

\author{
Katarina Monkova ${ }^{1, *}$, Peter Monka ${ }^{1,2}$, Jozef Tkac ${ }^{1}$ \\ ${ }^{1}$ Technical University of Kosice, Faculty of Manufacturing Technologies with the seat in Presov, \\ Sturova 31, 08001 Presov, Slovakia \\ ${ }^{2}$ Regional Technological Institute, University of West Bohemia, Univerzitni 8, 30614 Pilsen, Czech \\ Republic
}

\begin{abstract}
Direct metal laser sintering is spread technology today. The main advantage of this method is the ability to produce parts which have a very complex geometry and which can be produced only in very complicated way by classical conventional methods. Special category of such components are parts with porous structure, which can give to the product extraordinary combination of properties. The article deals with some aspects that influence the manufacturing of regular porous structures in spite of the fact that input technological parameters at various samples were the same. The main goal of presented research has been to investigate the influence of the volume ratio of solid phase on carrying capacity of regular porous structure. Realized tests have indicated that the unit of regular porous structure with lower volume ratio is able to carry a greater load to failure than the unit with higher volume ratio.
\end{abstract}

\section{Introduction}

Since the beginning of the 1980s additive manufacturing evolved from the first processes for the rapid production of prototypes into a number of different processes of which some are capable of direct part production. Today processes like selective laser melting (SLM), selective laser sintering (SLS) and with some limitations fused deposition modelling (FDM) are capable to produce direct parts in end-user quality out of metal or thermoplastics. Additive manufacturing processes are technologically specified for industrial production and due to a rising competition between service providers additive manufacturing becomes economically feasible for a growing number of industrial and enduser applications. [1]

Metal AM is an 'additive' technology that works by fusing together very fine layers of metal powder using a focused laser beam. This powder fusion process can produce complex geometries which might not have been possible using traditional manufacturing techniques. The process begins by slicing the CAD model to obtain a $2 \mathrm{D}$ contour at each level of the build axis, which is fixed as the z-axis. Starting from the base $2 \mathrm{D}$ contour, a user defined slice thickness is added cumulatively at successive slicing planes. This layer by layer

\footnotetext{
*Corresponding author: katarina.monkova@tuke.sk
} 
stacking gives rise to an error called the staircase effect which diminishes the surface finish of the part. It is the preferred method of $3 \mathrm{D}$ rapid prototyping for complex geometries that allows users to reduce the time and cost by completing multiple prototypes directly from CAD data. From a design perspective, the challenge of additive manufacturing is to understanding the limitations and opportunities of these new processes and to use them in the right applications. [2-4]

Scientists at the Massachusetts Institute of Technology have discovered new ways to use complex geometry to make strong, lightweight shapes that might be useful as structural materials for airplanes, automobiles and buildings. Authors' research deals with producing one type of regular porous structures - so called gyroid structure. [5]

\section{The samples of regular porous structure - gyroid}

Generally, porous structures are divided into two categories: regular and unregular - also called foams. The regular structures are better controlled in of mechanical engineering industry. Basic building units of geometrically defined porous structures are cells that are created by simple bodies, e.g. cube, cuboid, cylinder or sphere. These geometries are usually well modelled in CAD applications. More complex structures have their geometries created by curved surfaces. The scientists have found an inspiration related to the regular porous structure in nature. Many natural structures such as bone and some corals are built from strong microstructure building blocks. Using microscopes with three-dimensional nanoscale resolution, Yale University researchers found that shades of green in the wings of five butterfly species are produced by crystalline structures called gyroids. In the Fig. 1 is presented wing scale photonic nanostructure that was base of three steps towards to the virtual 3D model creation. $[6,7]$

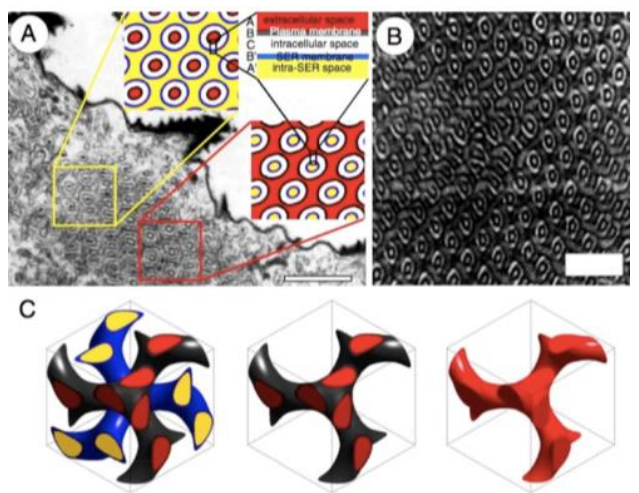

Fig. 1. Wing scale photonic nanostructure, from electron microscope to model. [7]

The shape of Shoen gyroid structure are described by mathematical formula (1) [8]

$$
\sin \left(\frac{2 \pi}{L} x\right) \cos \left(\frac{2 \pi}{L} y\right)+\sin \left(\frac{2 \pi}{L} y\right) \cos \left(\frac{2 \pi}{L} z\right)+\sin \left(\frac{2 \pi}{L} z\right) \cos \left(\frac{2 \pi}{L} x\right)-t=0,
$$

where factor $L$ influences the size of basic cell and parameter $t$ decides about cross section of the strut, what influences the relative density of structure. Next parameter - volume ratio $V r$ of solid phase expresses how many percent of the cell space is filled by material. It is given by equation (2): [9]

$$
V_{r}=\frac{\text { Volume of solid phase }}{\text { Total volume }} 100 \quad[\%]
$$


The samples for testing were produced by DMLS technology using EOS M290 machine with default technological parameters (laser power $400 \mathrm{~W}$, diameter of laser beam $100 \mu \mathrm{m}$ ). Material of samples was the Maraginsteel MS1. The particles of the powder were spherical with the diameters in the range of $15-50 \mu \mathrm{m}$. (Fig. 2) The difference in grain size is welcome, because smaller particles can fill in the space between bigger grains. $[10,11]$

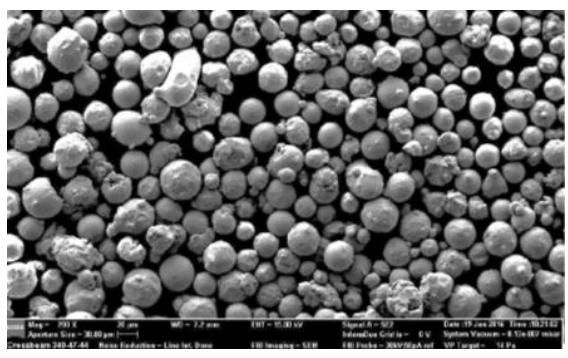

Fig. 2. Metal powder of Maraginsteel MS1 [10]

Conventional steel 1.2709 according to the European Standard has excellent mechanical properties, high yield strength and tensile strength, is stretchable and resistant to dynamic stress. In the case of additively sintered material, it has reduced ductility and resistance to dynamic stress. It can be cured with a simple heat treatment to an approximate hardness of about 54 HRC. Table 1 provides the chemical composition of the starting material of the EOS Maragingsteel MS1 powder. [12]

Table 1. Chemical composition of EOS Maragingsteel MS1 [12]

\begin{tabular}{|c|c|c|c|c|c|c|c|c|c|c|}
\hline Component & $\mathbf{F e}$ & $\mathbf{N i}$ & $\mathbf{C o}$ & $\mathbf{M o}$ & $\mathbf{T i}$ & $\mathbf{A l}$ & $\mathbf{C r}, \mathbf{C u}$ & $\mathbf{C}$ & $\mathbf{M n}, \mathbf{S i}$ & $\mathbf{P}, \mathbf{S}$ \\
\hline$[\%]$ & remnant & $17-19$ & $8.5-9.5$ & $4.5-5.2$ & $0.6-0.8$ & $0.05-0.15$ & $\leq 0.5$ & $\leq 0.03$ & $\leq 0.1$ & $\leq 0.01$ \\
\hline
\end{tabular}

Preliminary experiments have been aimed at assessing the ability of additive technology to print gyroid structures without supporting structures. All the samples were designed by means of software Siemens NX 10 CAD, where they were edited and adapted to additive manufacturing.

The specimens with basic cell, sizes ranging from $3 \mathrm{~mm}$ to $8 \mathrm{~mm}$ with a volume of $15 \%$, were printed. All these samples (with constant volume ratio of $15 \%$ ) were printed without defects, thereby confirming the self-supporting property of Gyroid within a given range of sizes. Similar results were obtained by [13], whose production equipment was able to print the smallest aliquots of the tested series, having a cell size of $2 \mathrm{~mm}$ and a volume of $15 \%$.

In this phase of preliminary experiments, next set of samples with constant cubic cell size of $6.5 \mathrm{~mm}$ and volume ratios $13.5 \%, 9.5 \%, 7.3 \%, 5.8 \%, 5.0 \%$ and $4.2 \%$ were printed. They are shown in Fig. 3. [14]
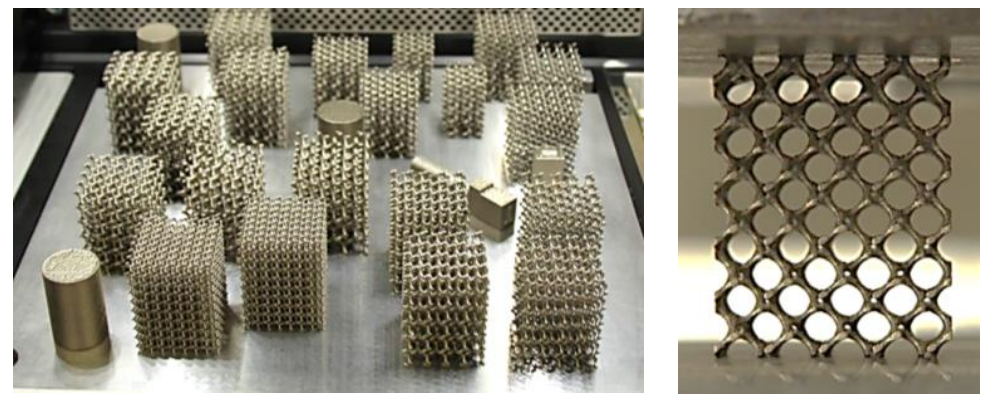

Fig. 3. Set of test specimens and the sample with cubic cell size of $6.5 \mathrm{~mm}$, volume ratio $9.5 \%$ [14] 
However, this preliminary experiment had some specifications. There have been some problems with deformation of samples during their production or during preliminary tests, which were necessary to solve.

\section{Some problems of samples production}

The first problem was related to the fact that some porous structures have deformed near the surface, where the sample was separated from a platform, see Fig. 4. [14]

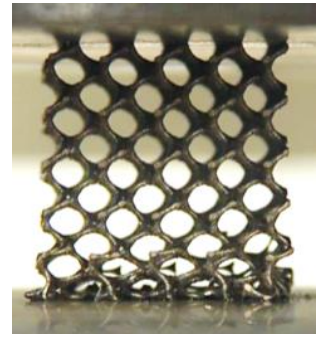

Fig. 4. Deformation of samples during preliminary pressure tests [14]

It was the reason why the basic structure (a deformation core) of sample has been equipped with two $2.5 \mathrm{~mm}$ high contact plates on both joining surfaces. The insertion of the end struts should support deformation throughout the porous structure during pressure tests to avoid local deformity at the point of contact with the test device head. Parallelism of the bearing surfaces should be ensured. A sample model of the test body from perspective is shown in Figure 5.

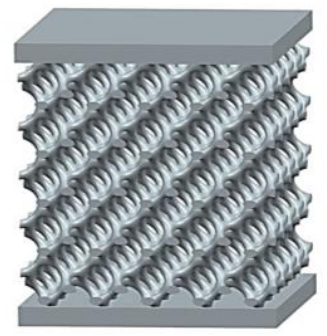

Fig. 5. The model of sample with bottom and top plate of continuous solid material

Figure 6a shows a model of a test body whose self-supporting function at the edges was disturbed. The deformation near the area of separation could be caused by stiffness reduction due to bending of strut structure during the cutting of sample with a band saw. To solve this problem, in relevant direction next one cell to the basic matrix of the sample was added and the sample was cut from support so to be its self-supporting function was kept. The sample with fully self-supporting function is shown in (Fig. 6b). [15]

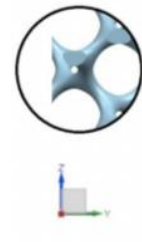

a)

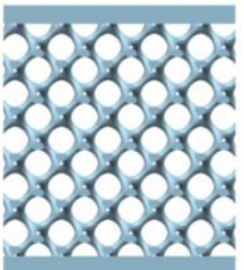

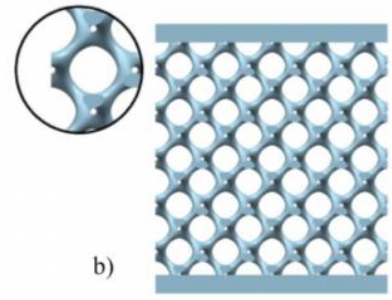

b)

Fig. 6. Self-supporting function of samples, a) disturbed, b) kept [15] 
In next phase of preliminary experiments the effect of orientation of gyroid structure on the machinability was investigated.

The lack of practical knowledge gave rise to another experiment in which the various orientations of the pressure samples during the additive production were evaluated. Figure 7 shows different position variations. The sample in Fig. 7a was printed in the vertical position. Subsequent rotation around the $y$ axis by $5^{\circ}$ was given a position for another sample shown in Fig. 7b. Larger rotation of the sample would considerably complicate the design of the support structures, as some portions of the porous core struts would need to be deposited with the support elements so that these critical parts did not begin to build in the bulk base of the production powder, resulting in defects in the porous core construction.

The last variant was produced in a horizontal position, see Fig. 7c. The specimen was extended by one row of cells at the point of contact with the platform to provide sufficient space for the cut during separation of the samples from the platform by the band saw. After cutting, the excess struts were manually cut off so that samples with a cubic porous core could originated. [14]

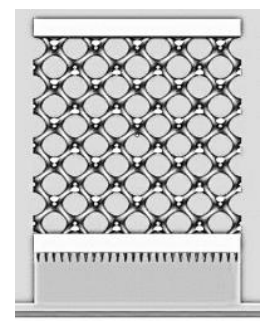

a)

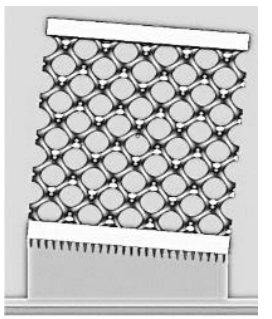

b)

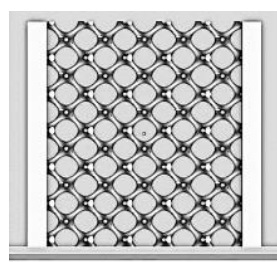

c)

Fig. 7. Orientation of tested samples [14]

Due to the fact that the structures positioned in version c), at which the continuous layers of material were on sides of structure (on left and right sides), had no imperfections, they were smooth and reliable in every manufacturing repetition, the horizontal position of sintering was evaluated as the most suitable for samples production, as it is displayed in Fig. 8.

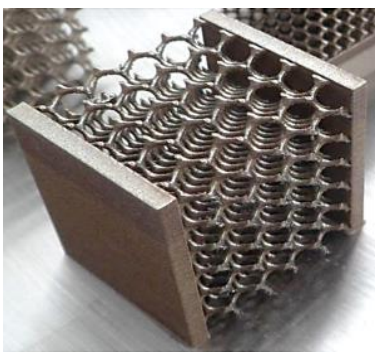

Fig. 8. Horizontal position as the best position for structure production

\section{Experiments}

The goal of test was the investigation of influence volume ratio on carrying capacity. Based on preliminary experiments, the sample size with five cells in one direction was set for experiments, because of the balance between the accuracy of the measured values and the economic indicator (the production cost per unit of sample). In the Fig. 9 is presented the gyroid structure at the same cell size, but with various volume ratios. 

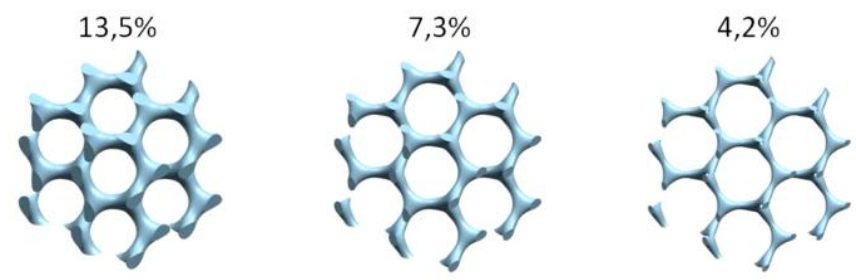

Fig. 9. The gyroid structure with various volume ratio

The samples were loaded up to the pressure that after exceeding the yield point of the material causes the irreversible plastic deformation. The test is finished if the set compression is achieved, or if the sample appeared significantly destructive conditions. The deformation characteristics, such are yield strength and maximum power load or load capacity have been evaluated from pressure diagram. Equipment Zwick/Roell Z250 with force capacity $250 \mathrm{kN}$ was selected for the test. The test specimens must not be subjected to a pressure deformation prior to the test. However, overloading may be necessary to eliminate clearance between the test and the test body, so the test samples were preloaded with a force of $10 \mathrm{~N}$. The compression speed of samples was $0.1 \mathrm{mms}^{-1}$ at the environment temperature of $20^{\circ} \mathrm{C}$. The results of tests are presented in Fig. 10. [14]

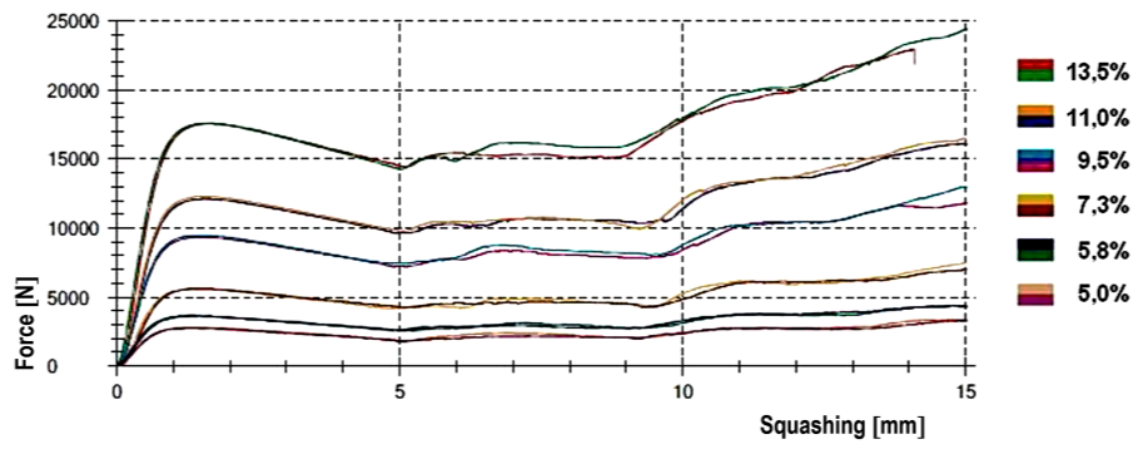

Fig. 10. The course of the deforming curves in dependence on the volume [14]

It is clear from the graphs displayed above that the relative carrying capacity of samples is slightly rising with volume ratio decreasing. It means that the unit of gyroid with lower volume ratio is able to carry a greater load to failure then the unit of gyroid structure with higher volume ratio.

\section{Conclusion}

Direct metal laser sintering is the preferred method of 3D rapid prototyping for complex geometries that allows users to reduce the time and cost by completing multiple prototypes directly from $\mathrm{CAD}$ data. From a design perspective, the challenge of additive manufacturing is to understanding the limitations and opportunities of these new processes and to use them in the right applications. [16]

Authors in the article have tried to point on some effects that influence the manufacturing of regular porous structures in spite of the fact that input technological parameters were set the same as the default parameters of the machine. These parameters are: geometry, slope of the sample and its positioning during laser sintering. Realized pressure tests also have indicated that the unit of gyroid with lower volume ratio is able to carry a greater load to failure than the unit of gyroid structure with higher volume ratio. 
The present contribution has been prepared under projects VEGA 1/0614/15, KEGA 087TUKE4/2015, KEGA 007TUKE-4/2018 and LO1502 'Development of the Regional Technological Institute' under the auspices of the National Sustainability Programme I of the Ministry of Education of the Czech Republic aimed at supporting research, experimental development and innovation.

\section{References}

1. D. Paramita et al.:, Procedia Manufacturing, 1, 343 - 354 (2015)

2. J. Dobransky, et al.:, Key Engineering Materials, 669, 532 - 540 (2016)

3. M, Cloot et al.: Assessing new support minimizing strategies for the additive manufacturing technology SLM. Solid Freeform Fabrication Symposium (2013)

4. S. M. Hur et al.: Determination of fabricating orientation and packing in SLS process. Journal of Material Processing Technology 236 - 243 (2001)

5. Beno, P., Kozak, D., Konjatic, P.:, Tehnicki Vjesnik, 5(21), 1051 - 1055 (2014)

6. F. Ferroudji et al.,Inter. J.of Mechanical \& Mechatronics Engineering IJMME/ IJENS, 04, 92 - 97 (2014)

7. S. H. Masood, W. Rattanawong, Int. J. of Advance Manuf. Technol., 19, 209 - 216 (2002)

8. V. Majstorovic et al., Procedia CIRP, 25, 27 - 32(2014)

9. D. T. Pham et al., Int. J.of Advance Manufacturing Technology, 15, 674 - 682 (1999)

10. P. Baron, et al., J. of the Inter. Measurement Confederation, 94, pp. 245 - 253 (2016)

11. S. Allen, D. Dutta, Solid Freeform Fabrication Symposium, Austin, 259-269 (1994)

12. A. Panda, et al.: Monitoring and Evaluation of Production Processes: An Analysis of the Automotive Industry, Springer International Publishing (2016)

13. K. Monkova, et al., Advances in Mechanical Engineering, 1-11 (2014)

14. P. Hanzl, M. Zetek, I. Zetkova:, Proceedings of the 26th DAAAM, 748 - 752 (2015)

15. P. Hanzl, I. Zetkova, J. Mach, Manufacturing Technology, 1, 34 - 38 (2017)

16. M. Ungureanu et al., Procedia Engineering, 149, 495 - 500 (2016) 
